\title{
Differential patterns of diversity at microsatellite, MHC, and TLR loci in bottlenecked South Island saddleback populations
}

\author{
Gabrielle J. Knafler $^{1 *}$, Catherine E. Grueber ${ }^{1,2,3}$, Jolene T. Sutton ${ }^{1,4}$ and Ian G. Jamieson ${ }^{1, \uparrow}$ \\ ${ }^{1}$ Department of Zoology, University of Otago, PO Box 56, Dunedin 9054, New Zealand \\ ${ }^{2}$ School of Life and Environmental Sciences, Faculty of Veterinary Science, University of Sydney, NSW, Australia \\ ${ }^{3}$ San Diego Zoo Global, San Diego, CA, USA \\ ${ }^{4}$ University of Hawai'i at Hilo, Hilo, HI, USA \\ ${ }^{\dagger}$ Deceased (2 February 2015) \\ *Author for correspondence (Email: dinodna1859@gmail.com)
}

Published online: 30 September 2016

\begin{abstract}
For populations that experienced historical population bottlenecks, subsequent contemporary bottlenecks may continue to degrade genetic diversity at loci that are still variable. However, it is currently unclear how different types of contemporary bottlenecks may affect diversity at functional versus neutral loci. In this study, we examine genetic diversity of microsatellite, major histocompatibility complex (MHC), and toll-like receptor (TLR) loci of three New Zealand South Island saddleback (Philesturnus carunculatus) populations: Big Island, Kaimohu Island and Motuara Island. One population, Motuara, experienced two sets of contemporary bottlenecks (translocation event followed by a disease-outbreak and population crash) and was sampled in three different years. For all populations and sampling periods, we compare patterns of diversity across the three sets of loci to infer the effects of contemporary bottlenecks. We found that translocation-induced bottlenecks resulted in greater losses of microsatellite than MHC diversity, whereas temporal (encompassing a potential disease-induced bottleneck) fluctuations in diversity were greater for MHC markers than microsatellite loci. Both translocation- and disease-induced contemporary bottlenecks affected TLR diversity to a greater extent than diversity of either microsatellites or MHC. We found significant temporal changes in the frequencies of two TLR1LA alleles on Motuara Island (coincident with the population crash), but no further subsequent change. These alleles differ by an amino acid variant (associated with the leucine-rich repeat region of TLR1LA). Since TLR1LA may initiate an innate immune response in the presence of haemosporidia parasites, it is possible that a selection event has contributed to the observed patterns at TLR1LA. Our results indicate that patterns of genetic diversity loss at microsatellite, MHC and TLR loci are not equally affected by contemporary population bottlenecks, and may be influenced by bottleneck type.
\end{abstract}

Key words: bottleneck; immune genes; pathogen-mediated selection

\section{Introduction}

Whether contemporary bottlenecks have significant effects on the genetic diversity of threatened populations largely depends on the history of the declining organism. It has been shown that factors such as location of the population (insular or mainlandoriginating), bottleneck severity (duration and intensity) and prior history of bottlenecks, are all strong determinants of remaining genetic diversity in threatened species (e.g. Taylor \& Jamieson 2008; Sutton et al. 2015). However, it is currently unknown whether population bottleneck circumstance (e.g. movement of animals by humans [i.e. translocations] vs. disease-induced) is important for driving genetic diversity outcomes in threatened populations when considering different molecular markers used to assess variation.

Anthropogenic practices, such as the movement of animals for conservation purposes, have served to re-establish or bolster populations that are in danger of becoming extinct (Griffith et al. 1989). Although translocations are a classic solution given the urgency of action required when an organism is declining in number (Griffith et al. 1989), there may be detrimental effects of inducing contemporary bottlenecks in already vulnerable species. It is commonly reported that translocation events contribute to the degradation of remaining genetic diversity in historically bottlenecked populations (Stockwell et al. 1996; Miller \& Lambert 2004; Lambert et al. 2005; Olson et al. 2013), although this depends on the extent to which genetic diversity has already been depleted (Taylor \& Jamieson 2008) (i.e. the variation at some genetic markers may be sufficiently depleted that there is none left to lose).

While anthropogenic practices are common causative agents for population bottlenecks of threatened species, natural processes such as disease outbreaks may induce further population declines (Hale \& Briskie 2009; Alley et al. 2010; Ujvari \& Belov 2011). In the case of disease, effects on genetic diversity at functional loci may differ from effects on neutral genetic diversity due to selective forces. For example, significant temporal changes in allele frequencies at loci important for immune function (the major histocompatibility complex, MHC) have been found in a migratory passerine, the great reed warbler (Acrocephalus arundinaceus), despite microsatellite loci maintaining stable allele frequencies (Westerdahl et al. 2004). In contrast, neutral processes, rather than selection, were responsible for shaping spatio-temporal MHC diversity in the Seychelles warbler (Wright et al. 2014), a historically bottlenecked isolated island species. However, we are aware of no studies that describe if/how the genetic diversity of a historically bottlenecked avian species changes 
over a time period that encompasses a further, disease-related population bottleneck. Here, we investigate spatial and temporal changes in the genetic diversity of New Zealand's South Island saddleback (Philesturnus carunculatus) by quantifying and comparing diversity of neutral microsatellite, adaptive immunity-associated MHC, and innate immunityassociated toll-like receptor(TLR) loci. We examine the impact of contemporary bottlenecks associated with human actions (specifically, translocation events) as well as a bottleneck caused by a disease outbreak, across all three genetic marker types.

South Island saddlebacks were widely distributed throughout New Zealand on the mainland of the South Island as well as on Stewart Island and a number of offshore islands before the introduction and spread of mammalian predators, specifically rats (Hoosen \& Jamieson 2003). By the early 1900 s, South Island saddlebacks were nearly extinct from the mainland and were limited to Big South Cape Island. After the invasion of rats onto this previously predator-free haven and subsequent saddleback extinction, 21 of the remaining South Island saddlebacks were transferred to Big Island and 15 were transferred to Kaimohu Island in 1964. Subsequently, a series of management translocations scattered South Island saddlebacks across several of New Zealand's offshore-islands, including the introduction of 19 birds from Big Island and Kaimohu Island to North Island (off the northeast coast of Stewart Island, Foveaux Strait) in 1972. The North Island population steadily grew and in 1994, 26 individuals were introduced from North Island to Motuara Island (Fig. 1a). By 2001, there were approximately 130 birds inhabiting Motuara Island (Hoosen \& Jamieson 2003). However, this population subsequently experienced a sudden and dramatic population crash (to 60 birds in March 2002) and then rebounded (>130 birds in 2005) (Hale \& Briskie 2009; Fig. 1b). The 2002 Motuara Island population bottleneck is believed to have been caused by a combination of pathogens including coccidian, Plasmodium spp. haemoparasites (i.e. avian malaria), and avipox virus (Hale \& Briskie 2009; Alley et al. 2010).

Previous studies indicate that South Island saddleback diversity at microsatellite loci was historically degraded and that no further losses of genetic diversity occurred through sequential contemporary translocations (Taylor \& Jamieson
2008). Despite contemporary populations of South Island saddlebacks also having reduced MHC diversity compared to historical populations (Sutton et al.2015), it is currently unclear whether diversity at functional genes has been sufficiently depleted to make the genetic effects of contemporary bottlenecks negligible. However, given the highly polymorphic state of the MHC and, to some extent, TLR loci in wild populations, we predict that (1) MHC and TLR loci will continue to decrease in diversity from source to serially translocated populations, and (2) neutral microsatellite diversity may vary slightly from 2002 to 2012 on Motuara Island due to the effects of genetic drift, while MHC and TLR diversity will show changes from 2002 to 2012 that reflect selective responses to the diseaserelated population bottleneck in 2004. This is the first study to investigate how contemporary bottlenecks affect the genetic diversity of two immune gene families, the MHC and TLRs, as well as putatively neutral microsatellite markers in a historically bottlenecked wild population.

\section{Materials and methods}

\section{Sampling}

South Island saddleback blood samples were collected from Big $(n=33)$ and Kaimohu $(n=16)$ Islands in 2005 (hereafter referred to as SB and SK for Big Island and Kaimohu Island populations, respectively). Motuara Island samples were collected in November $2002(n=30), 2004(n=30)$ and $2012(n=33)$ (hereafter SM2002, SM2004 and SM2012, respectively). Note that while we classify the Motuara samples separately and refer to them as 'populations' for convenience, our classifications are temporal rather than spatial and would allow for the possibility that individual birds may have existed across sampling records. However, due to the unique banding of all captured birds, we were able to deduce that there was no overlap in our sampled temporal populations. SB, SK $\left(1^{\text {st }}\right.$ order translocation), and SM2002 ( $3^{\text {rd }}$ order translocation) are populations affected by translocations whereas SM2002, SM2004 and SM2012 are populations that also were affected by disease. Of the samples collected from Motuara Island in 1a.

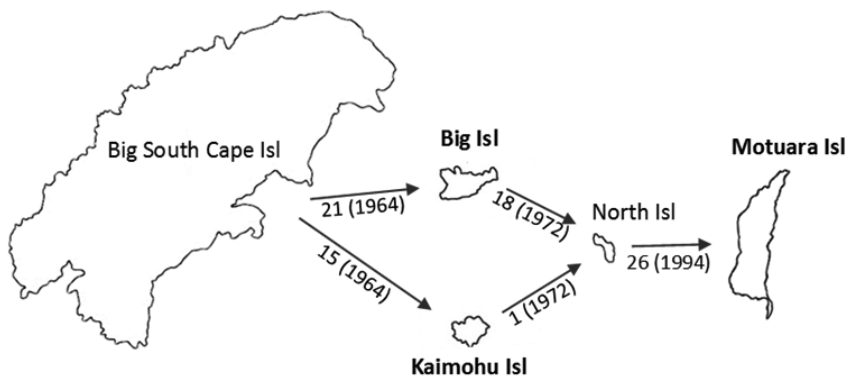

$1 \mathrm{~b}$.

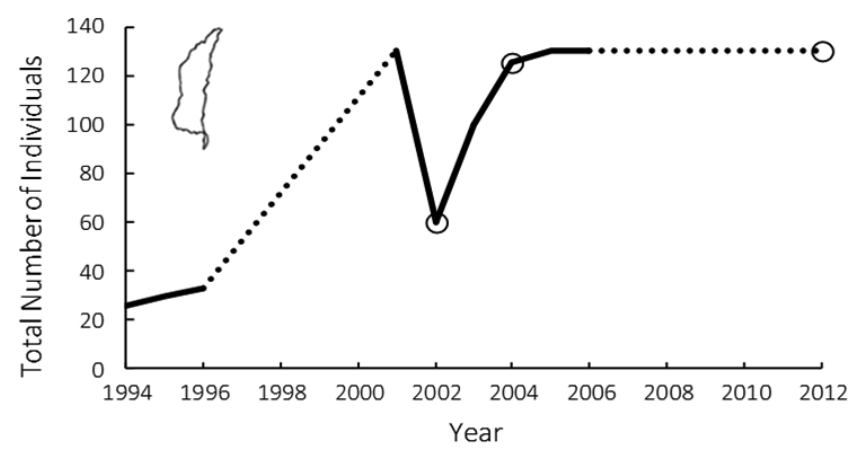

Figure 1a. Translocation history for South Island saddleback source (Big South Cape Island), first-order (Big and Kaimohu Islands; samples collected in 2005), second-order (North Island) and third-order (Motuara Island; samples collected in November 2002, 2004 and 2012) populations. Values outside and inside of parentheses represent the number of birds and year translocated at each stage, respectively. Islands for which we have samples are in bold. Locations of islands in relation to each other are not geographically accurate, although the scale is accurate. Figure 1b. Observed population growth of South Island saddlebacks on Motuara Island from introduction (1994) to most current population estimate (2012). The dotted lines represent periods when no population surveys were made, although field observations indicate the population was growing/stabilizing from 1997-2001 (Hale \& Briskie 2009) and from 2006 to 2012, respectively. Open circles represent years in which genetic sampling took place. 
November 2002-2004 and 2012, 70\% and 45\% were juveniles or sub-adults ( $<2$ years old), respectively, thus ensuring that we did indeed capture a portion of the next generation and not just a sub-sample of the original population. Genomic DNA was extracted from blood samples preserved at $4^{\circ} \mathrm{C}$ in Queen's lysis buffer (0.01 M Tris, 0.01 M sodium-EDTA, $0.01 \mathrm{M} \mathrm{NaCl}$, $1.0 \% n$-lauroylsarcosine, $\mathrm{pH} 8.0$; Seutin et al. 1991) using a $5 \%$ Chelex DNA extraction protocol (Walsh et al.1991).

\section{Microsatellite genotyping}

We amplified 18 microsatellite loci (see Table S1 in Supplementary Material) according to Sutton et al. (2015). Briefly, loci were amplified in $2 \mu$ multiplex reactions containing 10-15 ng of dried DNA, $1 \mu$ l Type-it Master Mix (Qiagen), and $1 \mu \mathrm{l}$ primer mix including $0.02-0.44 \mu \mathrm{M}$ forward primer with an M13 tail (5' - TGTAAAACGACGGCCAGT - 3'; Shuelke 2000), 0.08-1.76 $\mu \mathrm{M}$ reverse primer, and 0.16 $\mu \mathrm{M} \times \mathrm{N}_{\text {loci }}$ fluorophore-labelled (6FAM, VIC, PET, NED; Applied Biosystems DS-33) M13 universal primers (the same dye was consistently used for each locus to avoid scoring error, see Sutton et al. 2011). The thermal cycler profile consisted of $95^{\circ} \mathrm{C}$ for $5 \mathrm{~min}$, followed by 30 cycles of $30 \mathrm{~s}$ at $95^{\circ} \mathrm{C}$, $45 \mathrm{~s}$ at $60^{\circ} \mathrm{C}, 30 \mathrm{~s}$ at $72^{\circ} \mathrm{C}$ and eight cycles of $30 \mathrm{~s}$ at $95^{\circ} \mathrm{C}$, $45 \mathrm{~s}$ at $53^{\circ} \mathrm{C}, 30 \mathrm{~s}$ at $72^{\circ} \mathrm{C}$, with a final extension step of 30 $\min$ at $60^{\circ} \mathrm{C}$. Individuals were scored with GeneMarker ${ }^{\circledR}$ (SoftGenetics) using GeneScan500 LIZ as a size standard. Evidence for null alleles and allelic dropout was explored using Micro-Checker (Van Oosterhout et al. 2004). We tested for deviations from Hardy-Weinberg equilibrium and linkage disequilibrium with Arlequin v. 3.5.1.3 (Excoffier \& Lischer 2010). Utilizing microsatellite data, we inferred effective population size $(\mathrm{Ne})$ for populations of interest with ONeSAMP (Tallmon et al. 2008).

\section{MHC typing via next-generation sequencing}

We amplified (using primer pair PhcaMHCII.F.1 and PhcaMHCII.EX2.R.1; Sutton et al. 2013), purified, quantified, pooled, and sequenced a $240 \mathrm{bp}$ fragment of exon 2 from MHC class II B loci in all 142 individuals (plus 25 replicate samples) according to Knafler (2015). Briefly, a series of PCR reactions were carried out to attach unique DNA barcodes to all samples and achieve compatibility with Illumina's MiSeq flow cell. After quality and quantity of PCR products were estimated, all samples were pooled and sent to the University of Auckland to be sequenced using Illumina Miseq 2 X 150 bp chemistry (through NZ Genomics Ltd). Additionally, a portion $(n=8)$ of samples previously sequenced (Sutton et al. 2013) via Ion Torrent ${ }^{\mathrm{TM}}$ (Life Technologies; Rothberg et al. 2011) were re-sequenced in this study to control for different sequencing technologies on the detection of $\mathrm{MHC}$ sequence variants (hereafter referred to as MHC alleles for convenience, even though individual sequences could not be assigned to specific loci due to multiple loci co-amplifying from a single primer pair).

Initial MHC sequence data processing was achieved as outlined in Knafler (2015) except that reads were demultiplexed using an in-house PERL script (NZGL, personal communication) based on six in-line forward indices and 28 $\mathrm{R}$-adapter sequences. To validate sequences as 'true' alleles (i.e. separate from PCR and/or sequencing artefacts), we discarded all sequences that occurred below a minimum within-individual frequency threshold of $0.1 \%$. Threshold levels at $0.05 \%$, $0.3 \%, 0.5 \%, 1 \%, 5 \%$, and $10 \%$ were also considered. The
$0.1 \%$ threshold was chosen because increasing the threshold resulted in a loss of alleles independently detected in the Ion Torrent and Miseq sequencing experiments, while decreasing the threshold resulted in an increase in erroneous sequences found between replicate samples in this study. We discarded any sequences that were not found in replicate samples unless observed in at least two other individuals after calculating repeatability (Kennedy et al. 2002; Galan et al. 2010). We calculated repeatability in this study as 1-APD (average percent difference; Yuhki \& O'Brien 1990). We aligned alleles using BioEdit v 7.0.5.3 (Hall 1999). These alignments were then used to evaluate diversity statistics in DNAsp, including the total number of alleles $(A)$, number of polymorphic sites $(S)$, average number of nucleotide differences among all alleles identified in the population $(k)$, and nucleotide diversity $(\pi)$. The number of alleles per individual $(A / i)$ was calculated after allele identification.

\section{TLR typing via direct sequencing}

A portion (average of 14 individuals per population per locus) of all individuals were initially screened for diversity at toll-like receptor $1 \mathrm{LA}, 1 \mathrm{LB}, 2 \mathrm{~B}, 3,4,5$, and 15 variability at partial CDS regions (see Alcaide \& Edwards 2011; Grueber \& Jamieson 2013 for primer sequences; Grueber et al. 2015). The primers we used target exons encoding the leucine-rich repeat (LRR) regions of the extracellular domain of each TLR (Alcaide \& Edwards 2011; Grueber \& Jamieson 2013). Only if variation was present after initial screening were remaining individuals typed for that locus. Amplification of TLR segments was carried out in $15 \mu \mathrm{l}$ reactions containing 5-20 ng of DNA, $0.3 \mu \mathrm{M}$ of each primer, $1 \times$ MyTaq reaction buffer containing $3 \mathrm{mMMgCl}_{2}$ and $1 \mathrm{mM}$ of each $\mathrm{dNTP}$, and $0.6 \mathrm{U}$ of MyTaq polymerase (Bioline). The thermal cycling protocol (on an Eppendorf Mastercycler pro S) consisted of an initial denaturation of 3 min at $94^{\circ} \mathrm{C}$, followed by 35 cycles of $94^{\circ} \mathrm{C}$ for $40 \mathrm{~s}$, locusspecific annealing temperature (Grueber \& Jamieson 2013) for $40 \mathrm{~s}, 72^{\circ} \mathrm{C}$ for $80 \mathrm{~s}$, and a final extension of $72^{\circ} \mathrm{C}$ for 10 min. PCR products were excised from a $1 \%$ agarose gel and purified using the MEGA quick-spin ${ }^{\mathrm{TM}}$ total fragment DNA purification kit (iNtRON Biotechnology Inc.), and sequenced bi-directionally using the ABI BigDye Terminator version 3.1 on an ABI 3730xl DNAAnalyser (Genetic Analysis Services, University of Otago).

The program PHASE (Stephens et al. 2001; Stephens \& Donnelly 2003) implemented within DNAsp v 5.10.01 (Librado \& Rozas 2009) was used to reconstruct haplotypes from TLR direct sequencing files in which ambiguities represent polymorphic positions. We used the following MCMC default settings: number of iterations $=100$, thinning interval $=1$, burnin iterations $=100$ ). Diversity estimates, including the number of reconstructed haplotypes $(h)$, number of polymorphic sites $(S)$, average number of nucleotide differences $(k)$, nucleotide diversity $(\pi)$, and numbers of non-synonymous and synonymous SNPs were also obtained using DNAsp.

Observed and expected heterozygosity values $\left(H_{O}\right.$ and $H_{E}$, respectively), based on the inferred haplotypes, were calculated using Arlequin. Deviations from Hardy-Weinberg equilibrium were tested using Arlequin's HWE exact test with 1000000 steps in the Markov chain and 100000 dememorization steps. We employed LRR finder (Offord et al. 2010) to search for leucine-rich repeats and associated domains with positionspecific scoring matrix (PSSM) $=$ tlRRdb only with $95 \%$ and $80 \%$ upper and lower boundaries, respectively, within South Island saddleback TLR sequences aligned against chicken 
TLR reference sequences (see Results) to account for the fact that our primers do not amplify the full coding region of each gene (Grueber \& Jamieson 2013).

\section{Effects of bottlenecks on microsatellite, MHC and TLR markers}

Because the microsatellites used here are thought to be neutral with respect to natural selection, all observed microsatellite diversity patterns between SB, SK and SM2002 (translocated populations), and SM2002, SM2004 and SM2012 (putatively diseased populations) are assumed to be the result of genetic capture and/or genetic drift. Thus, comparisons among marker type can be used to infer whether neutral and adaptive/innate immunity-associated loci behave the same way in response to contemporary bottlenecks (Alcaide 2010).

We calculated pairwise $F_{\mathrm{ST}}$ values over all populations for microsatellite, MHC (by inputting sequences and the number of individuals carrying those alleles; Miller et al. 2010) and TLR markers in Arlequin. The relationship between pairwise microsatellite, MHC and TLR $F_{\mathrm{ST}}$ values was then assessed using a pairwise Mantel test implemented in GenAlEx v 6.5 (Peakall \& Smouse 2006, 2012) to identify if genetic differentiation between populations differs among marker type. We acknowledge that Jost's D and G' being used to delineate population differentiation using markers that describe non-adaptive events. However, there is not yet a standard way to calculate population differentiation for data in which there is co-amplification of duplicate loci. Therefore, we are following the protocol described in a similar study (Miller et al. 2010).

\section{Inferring contemporary selection on Motuara Island (MHC and TLRs)}

We tested whether observed fluctuations through time in allele frequencies (for TLRs) and proportion of individuals in which alleles occurred (for the MHC), were larger than expected by chance using permutation tests implemented in $\mathrm{R}$ ( $\mathrm{R}$ Core Team 2014). To test whether temporally characterised populations have indeed experienced a shift in genetic diversity (in contrast to artefactual shifts produced by sampling error), we employed a permutation test in which we obtained the distribution of the test statistic under the null hypothesis of no shift in genetic diversity by randomising the labels of the observed data and recalculating the change in diversity 1000 times. The proportion of times that the magnitude of the simulated change in allelic diversity was equal to (or greater than) the observed change(s) was recorded. If samples from two time periods do indeed represent distinct populations (i.e. the population has changed), there should be a small $(p \leq 0.05)$ probability of observing a simulated change of a similar or greater magnitude to the change(s) observed in the collected data.

We further modelled possible allele frequency changes (TLRs only) that may occur by genetic drift by using Monte Carlo simulations to model temporally-cultivated variation in allele frequencies (also in R;www.r-bloggers.com/simulatingallele-counts-in-a-population-using-r/, published 3 November 2013). The input parameters consisted of the number of simulations, starting frequency of the allele of interest, census and effective population sizes (analysed separately), and number of generations. The average of the simulated changes in allele frequency was computed and compared to the observed change(s) in allele frequency from the collected data.

\section{Results}

\section{Overall microsatellite diversity}

We removed all monomorphic microsatellite loci from further data analysis (13 of the 18 loci were fixed for one allele across all populations). The remaining polymorphic loci were 3B6, 6E4, Hru6, Ase18 and CK5 (Table 1). We did not identify any evidence for null alleles or allelic drop out. There were no significant deviations from Hardy-Weinberg equilibrium, nor evidence of linkage disequilibrium, at any locus in any population. Allelic richness averaged across loci (standardized to a sample size of 16) was 2.367, 2.175, 2.393, 2.397 and 2.183 for SB, SK, SM2002, SM2004 and SM2012, respectively (see Table 1 for allelic richness averaged across populations).

The minimum and maximum difference in microsatellite allele frequencies comparing Big Island to Kaimohu Island were 0.007 (locus CK5, allele 144) and 0.417 (locus Hru6, allele 226), respectively. Allele 251 (locus Ase18) was not found on Kaimohu Island (frequency on Big Island =0.212) and was lost over time on Motuara (present in 2002 and 2004, absent in 2012). The minimum and maximum magnitude of change in allele frequencies over time on Motuara Island were 0.00 (locus Hru6, allele 230, SM2002-SM2004) and 0.114 (locus 6E4, alleles 244/226, SM2002-SM2004), respectively. Effective population size on Motuara Island in 2002 based on microsatellite data was 20.019 (lower and upper 95\% CI = 12.697and 34.017, respectively).

\section{Overall MHC diversity}

We sequenced MHC class II B loci (240 bp after primer removal) in a total of 142 individuals (plus 25 replicate samples) using Illumina's MiSeq chemistry. We obtained an average of 14 823 reads per individual $(\mathrm{SD}=8866)$ after demultiplexing and quality trimming. All reported sequence variants were found across replicate samples (repeatability $=0.984$ ) or in at least two other individuals. Across all populations, we identified a total of 24 alleles. A phylogenetic tree indicated that 15 of the 24 identified alleles either fell into a cluster of putative non-classical sequences (exhibiting limited polymorphisms) or contained stop codons (see Figure S1 in Supplementary Material; Sutton et al. 2013). The total number of sequences per individual ranged from 11-23, potentially indicating the simultaneous amplification of 12 MHC class II B loci, a high value that is characteristic of passerine species (Bollmer et al. 2010). The number of classical alleles per individual ranged from four to nine; our main MHC analyses focused on these

Table 1. Mean allelic richness (AR), mean observed $\left(\mathrm{H}_{\mathrm{O}}\right)$ and expected $\left(\mathrm{H}_{\mathrm{E}}\right)$ heterozygosity with associated standard deviations in parentheses of all variable microsatellite loci averaged over five South Island saddleback populations ( $n$ $=142$ total individuals/locus).

\begin{tabular}{lccc}
\hline Locus & $\mathrm{AR}$ & $\mathrm{H}_{\mathrm{O}}$ & $\mathrm{H}_{\mathrm{E}}$ \\
\hline 3B6 & $2.000(0.000)$ & $0.363(0.088)$ & $0.377(0.084)$ \\
6E4 & $1.999(0.001)$ & $0.370(0.183)$ & $0.375(0.137)$ \\
Hru6 & $3.927(0.070)$ & $0.700(0.153)$ & $0.659(0.134)$ \\
Ase18 & $1.592(0.541)$ & $0.146(0.153)$ & $0.136(0.142)$ \\
CK5 & $1.999(0.002)$ & $0.334(0.076)$ & $0.313(0.061)$ \\
\hline
\end{tabular}


classical alleles. The proportion of individuals with each classical allele ranged from $0.06-1.00$ over all populations. The nine classical alleles, across all populations, showed 152 segregating sites $(S)$ and a nucleotide diversity $(\pi)$ of 0.15 .

Allele PhcaMHCII045 was fixed on Kaimohu Island (i.e. observed in all sampled birds) but not Big Island (proportion $=$ $0.824)$; and was observed at a similar proportion on Motuara Island in 2002 (0.857). The proportion of individuals that allele PhcaMHCII045 occurred in fluctuated through time on Motuara Island, first decreasing to 0.788 (SM2004, a decrease of 0.069 ) and then increasing to 0.912 (SM2012, an increase of 0.124). Another allele, PhcaMHCII047, also fluctuated in the Motuara population, starting at 0.905 (SM2002), but then increasing to 0.970 (SM2004, an increase of 0.065) before decreasing to 0.853 (SM2012, a decrease of 0.117).

\section{Overall TLR diversity}

We obtained an average of $1075 \mathrm{bp}$ of sequence for TLR1LA (1169 bp), 1LB (996 bp), 2B (1178 bp), 3 (1149 bp), 4 (621 bp), 5 (1167 bp), and 15 (1250 bp) from an average of 14 individuals per locus per population. TLR1LA was the only variable locus, therefore all remaining sampled individuals were sequenced and analysed for variation at this locus only (total $n$ across populations $=135$ ). We observed nucleotide variants at two positions (both nonsynonymous), resulting in four different nucleotide and amino acid sequences over all populations (Fig. 2). There were no significant deviations from Hardy-Weinberg expectations in any population except for Big Island (homozygote excess, $p=0.030$; Table 2).

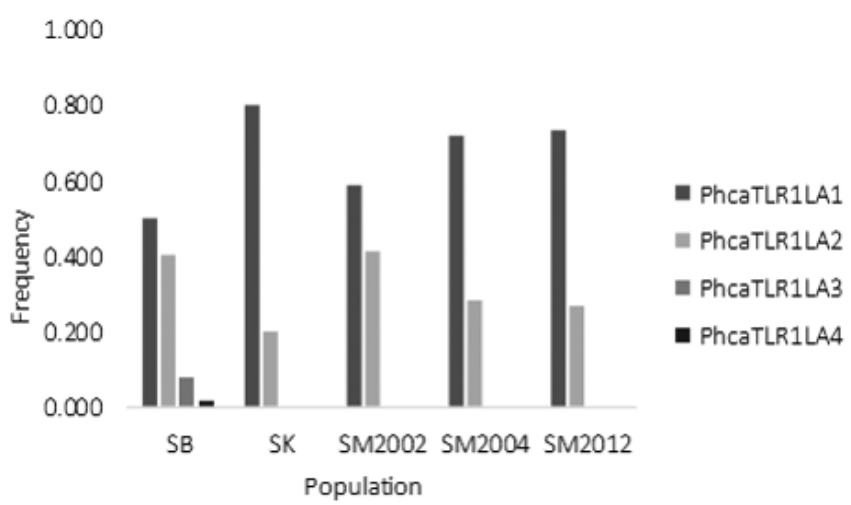

Figure 2. Frequency of TLR1LA alleles across five populations or South Island saddleback.

\section{Effects of bottlenecks on microsatellite, MHC and TLR markers}

Microsatellite pairwise $F_{\mathrm{ST}}$ analyses revealed moderate but significant structuring only between SK/SB $(0.144, p \leq 0.001)$ and SK/SM2002 $(0.068, p=0.009)$. We did not detect any population structure based on MHC loci. TLR1LA pairwise $F_{\mathrm{ST}}$ analyses revealed statistically significant moderate structuring between SK/SB $(0.100, p=0.027)$ and SM2002/ SM2012 $(0.039, p=0.036)$. See Table S3 in Supplementary Material for microsatellite, MHC and TLR pairwise $F_{\mathrm{ST}}$ values. Pairwise Mantel tests revealed no significant correlations for MHC/microsatellite $\left(\mathrm{R}^{2}=0.322, p=0.070\right)$ and TLR1LA/ microsatellite $\left(\mathrm{R}^{2}=0.015, p=0.390\right)$ pairwise $F_{\mathrm{ST}}$ comparisons (see Figure S2a in Supplementary Material). However, the MHC and TLR pairwise $F_{\mathrm{ST}}$ values were significantly negatively correlated $\left(\mathrm{R}^{2}=0.296, p=0.030\right.$, see Figure $\mathrm{S} 2 \mathrm{~b}$ in Supplementary Material), indicating that when pairs of populations differed more at the MHC, they differed less at TLR1LA.

\section{Assessing contemporary selection on Motuara Island (MHC and TLRs)}

Across temporal samples on Motuara Island (which encompass a disease-induced bottleneck; Fig. 1), the largest changes in MHC diversity that we observed occurred for the proportion of individuals that carried PhcaMHCII045 and PhcaMHCII047; all other alleles either remained constant or showed smaller fluctuations. Permutation tests indicated that the probability of observing an equal or greater magnitude change in the proportion of individuals carrying PhcaMHCII045 was $p=$ 0.159 and $p=0.951$ from 2002 to 2004 and from 2004 to 2012 , respectively (Table 3 ). Likewise, the probability of observing an equal or greater magnitude change in the proportion of individuals carrying PhcaMHCII045 was $p=0.942$ and $p=$ 0.080 from 2002 to 2004 and from 2004 to 2012, respectively (Table 3). Thus, the observed changes are consistent with chance fluctuations due to sampling error.

We observed a considerable change in the allele frequencies of the PhcaTLR1LA1 and PhcaTLR1LA2 sequence variants (magnitude of change $=0.133$ from Motuara Island 2002 to 2004; but negligible change from 2004 to 2012; Fig. 2). Permutation tests indicated that the probability of observing an equal or greater magnitude change in the frequencies PhcaTLR1LA1 and PhcaTLR1LA2 was $p=0.039$ and 0.016 , respectively (Table 3 ). Thus, the observed changes were unlikely to have occurred by chance via sampling error. Similarly, the average expected change in PhcaTLR1LA1 over two generations as modelled via Monte Carlo simulations (1000) was 0.042 (95\%

Table 2. TLR1LA diversity measures from $(n)$ individuals including the number of reconstructed haplotypes $(h)$, number of polymorphic sites $(S)$, numbers of synonymous (Syn) and non-synonymous (Nsyn) sites, observed $\left(H_{O}\right)$ and expected $\left(H_{E},\right)$ heterozygosities per population, and HWE test $\mathrm{p}$-value $(p)$.

\begin{tabular}{lcccccccc}
\hline Pop & $n$ & $h$ & $S$ & Syn & Nsyn & $H_{O}$ & $H_{E}$ & $p$ \\
\hline SB & 30 & 4 & 2 & 0 & 2 & 0.39 & 0.58 & 0.03 \\
SK & 15 & 2 & 1 & 0 & 1 & 0.40 & 0.32 & 1.00 \\
SM2002 & 29 & 2 & 1 & 0 & 1 & 0.33 & 0.39 & 0.70 \\
SM2004 & 30 & 2 & 1 & 0 & 1 & 0.55 & 0.49 & 0.67 \\
SM2012 & 31 & 2 & 1 & 0 & 1 & 0.38 & 0.40 & 0.38 \\
\hline
\end{tabular}


Table 3. Results from the Motuara Island permutation tests. Year indicates the time period in which observed changes (Obs. change; proportion of individuals and frequency of alleles for MHC and TLRs, respectively) took place. The proportion of times the expected change in allelic diversity was equal to or greater/less than the observed change is indicated by $p$. Negative observed change values indicates a decrease in the proportion of individuals a particular allele occurred in over the specified time range. Significant $p$-values $(p \leq 0.05)$ are in bold.

\begin{tabular}{ccccc}
\hline Gene & Allele & Year & $\begin{array}{c}\text { Obs. } \\
\text { change }\end{array}$ & $p$ \\
\hline \multirow{2}{*}{ TLR1LA } & PhcaTLR1LA1 & $2002-2004$ & 0.133 & $\mathbf{0 . 0 3 9}$ \\
& & $2004-2012$ & 0.014 & 0.490 \\
& PhcaTLR1LA2 & $2002-2004$ & -0.133 & $\mathbf{0 . 0 1 6}$ \\
& & $2004-2012$ & -0.014 & 0.487 \\
MHCII $^{1}$ & PhcaMHCII045 & $2002-2004$ & -0.069 & 0.159 \\
& & $2004-2012$ & 0.124 & 0.951 \\
& PhcaMHCII047 & $2002-2004$ & 0.065 & 0.942 \\
& & $2004-2012$ & -0.135 & 0.080 \\
\hline
\end{tabular}

${ }^{1}$ Analysis for MHC was only conducted on the two alleles that showed the greatest magnitude of change.

$\mathrm{CI}=0.039$ and 0.045$)$ when using an effective population size of 20 individuals and starting allele frequency of 0.058 . Aligning PhcaTLR1LA1 against chicken toll-like receptor 1 (Gallus gallus; GenBank Accession NP_001007489; see Figure S3 in Supplementary Material) indicated that the amino acid substitution from Serine to Tyrosine, which differentiates PhcaTLR1LA1 and PhcaTLR1LA2, is located within the leucine-rich repeat region at position 234 in the chicken reference sequence. When comparing our data to TLR1LA sequences across 20 other avian species (Grueber et al. 2014), this position is also variable (Serine/Proline/Alanine).

\section{Discussion}

In this study, we examined patterns of genetic diversity at microsatellite, MHC, and TLR loci within and across three South Island saddleback populations (Big Island, Kaimohu Island, and Motuara Island (the latter site was sampled in 2002, 2004, and 2012)) to identify how contemporary bottlenecks affected different loci. We interpreted these observations in the context of anthropogenic- (associated with translocation events) and disease-induced bottlenecks, and their impacts on diversity loss across loci. In part, our hypotheses were confirmed: (1) TLR loci decreased in diversity from source to translocated populations, whereas the MHC was less affected and (2) TLR1LA showed considerable temporal diversity changes on Motuara Island, potentially due to an immunogenetically-related selection response, which were not reflected at microsatellites or the MHC.

\section{Effects of bottlenecks on microsatellite, MHC and TLR markers}

Significant population differentiation (based on $F_{\mathrm{ST}}$ values) was only observed among translocated populations (and not among temporal samples on Motuara) suggesting that anthropogenic-associated bottlenecks played a greater role in shaping microsatellite diversity patterns than bottlenecks associated with stochastic events such as the Motuara Island 2002 pathogen-associated population crash. Allele frequencies at variable microsatellite loci differed considerably on Big Island compared to Kaimohu Island, likely due to anthropogenic sampling effects (note that one portion of known remaining South Island saddlebacks was transferred to Big Island and the other portion was translocated to Kaimohu Island; Taylor \& Jamieson 2008). In contrast, microsatellite allelic frequencies on Motuara Island in 2002 mirrored that of Big Island, likely because only one individual was transferred from Kaimohu to North Island (the source population for Motuara Island) while 18 were transferred from Big to North Island (Fig. 1a). It is worth noting that rapid population expansions have been shown to produce complex diversity patterns at neutral genetic loci (Excoffier \& Ray 2008), and therefore may be a contributing factor to our observed structuring, although we do not have detailed information regarding saddleback range expansion on these islands that would be required to test this hypothesis.

Unlike microsatellite markers, there was no significant differentiation at MHC loci, among any populations as evidenced by $F_{\mathrm{ST}}$. Like microsatellite markers, MHC allelic proportions on Motuara Island 2002 mirrored those of Big Island. Over time, MHC alleles did vary in their frequencies; some changes were even greater than those observed for microsatellite alleles, but permutation tests indicated that the observed changes were not significantly different from those expected by chance alone (Table 3 ). Thus, sampling effects could be responsible for the observed changes in MHC diversity (we did not sample the entire population on the island at each time point).

Like microsatellites, but unlike MHC markers, there was significant structuring among Big and Kaimohu Islands as identified by $F_{\mathrm{ST}}$ values for TLRs. However, unlike both microsatellite and MHC markers, TLR allele frequencies changed significantly between SM2002 and SM2012 (based on $F_{\mathrm{ST}}$ and permutation tests), suggesting that both anthropogenic and natural bottlenecks affected TLR diversity patterns in these populations. Furthermore, unlike microsatellites and MHC, TLR1LAwas the only locus that deviated from Hardy-Weinberg equilibrium, showing a homozygote excess on Big Island. This deviation may be a signal of selection; however, we are currently unable to disentangle the effects of drift from selection in this 
population. Finally, the only significant relationship among all three marker types (considering pairwise $F_{\mathrm{ST}}$ comparisons across all populations) was a negative correlation between the MHC and TLRs (Figure S2), thus supporting the observation of differential diversity patterns across loci.

\section{Inferring contemporary selection on Motuara Island (TLRs)}

We found a significant increase and corresponding decrease in the frequencies of PhcaTLR1LA1 and PhcaTLR1LA2, respectively, from 2002 to 2004 on Motuara Island (Fig. 2). The observed TLR1LAallele frequency fluctuations were larger than the maximum observed microsatellite allele frequency fluctuations from 2002 to 2004 but not from 2004 to 2012 . Additionally, the average expected allele frequency change from 2002 to 2004 modelled via Monte Carlo simulations (assuming random genetic drift) was less than observed TLR1LA allele frequency changes. Thus, if PhcaTLR1LA alleles are involved in pathogen resistance, a selective response to the Motuara Island population bottleneck in 2002 may be partially responsible for the observed temporal patterns of TLR1LA (see review by Spurgin \& Richardson 2010). An alternative explanation for the large changes in TLR1LA allele frequencies from 2002 to 2004 on Motuara Island is a founder effect, especially given that the census population size dramatically declined approximately from 130 to 60 individuals ( $\mathrm{Ne}$ estimated to be 20). However, if the decrease in effective population size produced the observed changes in TLR allelic frequencies, then we would expect to see similar results at microsatellite loci and potentially the MHC. Neither microsatellite nor MHC frequency/proportion changes paralleled that of TLR1LA, providing support for the idea that the observed shifts in diversity may be due to natural selection.

The SM2002 population decline is believed to have been caused by a combination of pathogens including coccidian, Plasmodium spp. haemoparasites, and avipox virus (Hale \& Briskie 2009; Alley et al. 2010). In mammals, experiments have shown that TLR2 forms heterodimers with TLR1/TLR6/ TLR10 to recognize di- and tri-acylated lipoproteins/peptides (Buwitt-Beckmann et al. 2006). In birds, the dimerization of TLR2/TLR1LA allows for the recognition of the same broad array of molecules that the dimerization of TLR2/TLR1, 6 and 10 permits in mammals (Brownlie \& Allan 2011). Although di- and tri-acylated lipopetides are commonly associated with cell wall components of bacteria, the ultrastructure of avian Plasmodia generally consists of a pellicle composed of three membranes (Valkiūnas 2005) that may be the recognition source for TLR1LA. Furthermore, the amino acid substitution differentiating PhcaTLR1LA1 and PhcaTLR1LA2 is a nonconservative change from 'tiny' Serine to 'large hydrophobic' Tyrosine (Betts \& Russell 2007), and is located within the functional leucine-rich repeat region at position 234 (aligned against chicken reference sequence). Although amino acid changes of this type may influence the binding efficiency of the peptide, functional assays of the protein variants would be required to confirm this. When compared to TLR1LA sequences in other avian species (Grueber et al. 2014), substitutions at this positon result in Serine/Proline/Alanine amino acid changes that suggest its potential importance for pathogenassociated molecular pattern recognition. Nonetheless, it is important to note that since we have not sequenced the entire TLR1LA coding region, we cannot exclude the possibility that the functional variant(s) responsible for the putative selection event lie outside of the sequenced region.

\section{Cautions of this study}

We have attempted to highlight the difference in patterns of genetic diversity loss at neutral and functional loci under two different population bottleneck scenarios; specifically, anthropogenically bottlenecked populations (SB, SK and SM2002) and disease-induced population bottlenecks (SM2002, SM2004 and SM2012). Although we are treating the two population bottleneck scenarios as independent events, we note that the SM2002 population was founded by translocated individuals from Big and Kaimohu Islands. Thus, the contemporary disease-induced bottleneck on Motuara Island in 2004 was not the only contemporary bottleneck affecting the Motuara Island population. Furthermore, the genetic diversity estimates on Motuara Island in 2002 cannot be considered completely due to the third-order translocation (Fig. 1a) as the pathogen-associated bottleneck was in effect. Finally, because our study system is limited to wild populations, we were unable to control certain possible confounders such as putative pathogen-associated natural selection in the translocated island populations ( $\mathrm{SB}, \mathrm{SK}$ and $\mathrm{SM}$ prior to the known disease outbreak).

\section{Conclusion}

In summary, patterns of genetic diversity loss at microsatellite, MHC and TLR loci in New Zealand's South Island saddleback were not equal. Translocation-induced loss of alleles occurred at one microsatellite locus and no MHC loci, whereas temporal (encompassing a potential disease-induced bottleneck) fluctuations in diversity were greater for MHC markers than microsatellite loci. However, the observed microsatellite and MHC diversity patterns could be attributed to sampling (either natural (e.g. genetic drift) or anthropogenic (genetic capture)) effects rather than a selection event as microsatellite loci are putatively neutral and our analyses indicate that temporal MHC variation may be a sampling artefact. In contrast, both translocation- and disease-induced contemporary bottlenecks affected TLR diversity to a greater extent than MHC and microsatellites.

Population diversity of the only polymorphic toll-like receptor locus, TLR1LA, was variable across space and time. Two of the four TLR1LA alleles were lost most likely due to the movement of animals for conservation purposes (translocationinduced bottleneck). As opposed to microsatellite and $\mathrm{MHC}$ loci, changes in TLR1LA allele frequencies on Motuara Island appear to be the consequence of a selective response to a pathogen outbreak as evidenced by the fact that (1) significant changes in TLR1LA allele frequencies were observed from 2002 to 2004 but not from 2004 to 2012; (2) the observed TLR1LA allele frequency changes were larger than observed microsatellite allele frequency changes and larger than expected by chance; and (3) the non-conservative amino acid substitution differentiating the putatively selected alleles was located within the functional leucine-rich repeat region and was also variable across other avian species. Future studies could evaluate the functional significance of the observed variation in South Island saddleback TLR1LA and whether variation in the reported amino acids is truly associated with variation in resilience to pathogens of the sort implicated in the recent population crash on Motuara Island. Our study shows that variability in the genetic diversity of a historically bottlenecked species can still be detected and reflects the 
population-specific narrative of decline. Thus, we highlight the importance of continued discussion surrounding management of a threatened organism thought to be a 'lost cause' due to very low genetic diversity.

\section{Acknowledgements}

We are grateful to Gregory Gimenez, Les McNoe, and Monika Zavodna at Otago Genomics and Bioinformatics Facility for their valuable NGS advice. We thank Katrina Hale and Sabrina Taylor for help in collecting and providing samples. We also appreciate Dan Tompkins (Landcare Research) for providing comments on this manuscript and Ken Miller for assistance with Figure 1. This research was supported by an Allan Wilson Centre grant to IGJ and University of Otago scholarship to GJK.

\section{References}

Alcaide M 2010. On the relative roles of selection and genetic drift in shaping MHC variation. Molecular Ecology 19: 3842-3844.

Alcaide M, Edwards SV 2011. Molecular evolution of the toll-like receptor multigene family in birds. Molecular Biology and Evolution 28: 1703-1715.

Alley MR, Hale KA, Cash W, Ha HJ, Howe L 2010. Concurrent avian malaria and avipox virus infection in translocated South Island saddlebacks (Philesturnus carunculatus carunculatus). New Zealand Veterinary Journal 58: 218-223.

Betts MJ, Russell RB 2007. Amino acid properties and consequences of substitutions. In: Barnes MR ed. Bioinformatics for geneticists. 2nd edn. Hoboken, NJ, John Wiley \& Sons Ltd. Pp. 289-316.

Bollmer JL, Dunn PO, Whittingham LA, Wimpee C 2010. Extensive MHC class II B gene duplication in a Passerine, the common yellowthroat (Geothlypis trichas). Journal of Heredity 101: 448-460.

Brownlie R, Allan B 2011. Avian toll-like receptors. Cell and Tissue Research 343: 121-130.

Buwitt-Beckmann U, Heine H, Wiesmüller KH, Jung G, Brock R, Akira S, Ulmer AJ 2006. TLR1-and TLR6-independent recognition of bacterial lipopeptides. The Journal of Biological Chemistry 281: 9049-9057.

Excoffier L, Lischer HEL 2010. Arlequin suite ver 3.5: a new series of programs to perform population genetics analyses under Linux and Windows. Molecular Ecology Resources 10: 564-567.

Excoffier L, Ray N2008. Surfing during population expansions promotes genetic revolutions and structuration. Trends in Ecology and Evolution 23: 347-351.

Galan M, Guivier E, Caraux G, Charbonnel N, Cosson J-F 2010. A 454 multiplex sequencing method for rapid and reliable genotyping of highly polymorphic genes in largescale studies. BMC Genomics 11: 296.

Griffith B, Scott JM, Carpenter JW, Reed C 1989. Translocations as a species conservation tool: status and strategy. Science 245: 477-480.

Grueber CE, Jamieson IG 2013. Primers for amplification of innate immunity toll-like receptor loci in threatened birds of the Apterygiformes, Gruiformes, Psittaciformes and Passeriformes. Conservation Genetic Resources 5: 1043-1047.

Grueber CE, Wallis GP, Jamieson IG 2014. Episodic positive selection in the evolution of avian toll-like receptor innate immunity genes. PloS ONE 9: e89632.

Grueber CE, Knafler GJ, King TM, Senior A, Grosser S, Robertson B, Weston KA, Brekke P, Harris Christion LW, Jamieson IG 2015. Toll-like receptor diversity in 10 threatened bird species: relationship with microsatellite heterozygosity. Conservation Genetics 16: 595-611.

Hale KA, Briskie JV 2009. Rapid recovery of an island population of the threatened South Island saddleback Philesturnus c. carunculatus after a pathogen outbreak. Bird Conservation International 19: 239.

Hall TA 1999. BioEdit: a user-friendly biological sequence alignment editor and analysis program for Windows 95/98/ NT. Nucleic Acids Symposium Series 41: 95-98.

Hooson S, Jamieson IG 2003. The distribution and current status of New Zealand saddleback Philesturnus carunculatus. Bird Conservation International 13: 79-95.

Kennedy LJ, Ryvar R, Gaskell RM, Addie DD, Willoughby K, Carter SD, Thomson W, Ollier WE, Radford AD 2002. Sequence analysis of MHC DRB alleles in domestic cats from the United Kingdom. Immunogenetics 54: 348-352.

Knafler G 2015. The genetic effects of bottlenecks and disease in three iconic New Zealand bird species. Unpublished PhD Thesis, University of Otago, Dunedin. 152 p.

Lambert DM, King T, Shepherd LD, Livingston A, Anderson S, Craig JL 2005. Serial population bottlenecks and genetic variation: translocated populations of the New Zealand saddleback(Philesturnus carunculatus rufusater). Conservation Genetics 6: 1-14.

Librado P, Rozas J 2009. DnaSP v5: a software for comprehensive analysis of DNA polymorphism data. Bioinformatics 25: 1451-1452.

Miller HC, Lambert DM 2004. Genetic drift outweighs balancing selection in shaping post-bottleneck major histocompatibility complex variation in New Zealand robins (Petroicidae). Molecular Ecology 13: 37093721.

Miller HC, AllendorfF, Daugherty CH 2010. Genetic diversity and differentiation at MHC genes in island populations of tuatara (Sphenodon spp.). Molecular Ecology 19: 3894-3908.

Offord V, Coffey TJ, Werling D 2010. LRRfinder: a web application for the identification of leucine-rich repeats and an integrative toll-like receptor database. Developmental and Comparative Immunology 34: 1035-1041.

Olson ZH, Whittaker DG, Rhodes OE 2013. Translocation history and genetic diversity in reintroduced bighorn sheep. The Journal of Wildlife Management 77: 1553-1563.

Peakall R, Smouse PE 2006. GENALEX 6: genetic analysis in Excel. Population genetic software for teaching and research. Molecular Ecology Notes 6: 288-295.

Peakall R, Smouse PE 2012. GenAlEx 6.5: genetic analysis in Excel. Population genetic software for teaching and research - an update. Bioinformatics 28: 2537-2539.

R Core Team 2014. R: a language and environment for statistical computing. R Foundation for Statistical Computing. www.R-project.org/.

Richardson DS, Jury FL, Dawson DA, Salgueiro P, Komdeur J, Burke T 2000. Fifty Seychelles warbler (Acrocephalus sechellensis) microsatellite loci polymorphic in Sylviidae 
species and their cross-species amplification in other passerine birds. Molecular Ecology 9: 2225-2230.

Rothberg JM, Hinz W, Rearick TM, Schultz J, Mileski W, Davey M, Leamon JH, Johnson K, Milgrew MJ, Edwards M, Hoon J, Simons JF, Marran D, Myers JW, Davidson JF, Branting A, Nobile JR, Puc BP, Light D, Clark TA, Huber M, Branciforte JT, Stoner IB, Cawley SE, Lyons M, Fu Y, Homer N, Sedova M, Miao X, Reed B, Sabina J, Feierstein E, Schorn M, Alanjary M, Dimalanta E, Dressman D, Kasinskas R, Sokolsky T, Fidanza JA, Namsaraev E, McKernan KJ, Williams A, Roth GT, Bustillo J 2011. An integrated semiconductor device enabling non-optical genome sequencing. Nature 475: 348-352.

Spurgin LG, Richardson DS 2010. How pathogens drive genetic diversity: MHC, mechanisms and misunderstandings. Proceeding of the Royal Society B 277: 979-988.

Stephens M, Donnelly P 2003. A comparison of Bayesian methods for haplotype reconstruction from population genotype data. American Journal of Human Genetics 73: $1162-1169$.

Stephens M, Smith NJ, Donnelly P 2001. A new statistical method for haplotype reconstruction from population data. American Journal of Human Genetics 68: 978-989.

Stockwell CA, Mulvey M, Vinyard GL 1996. Translocations and the preservation of allelic diversity. Conservation Biology 10: 1133-1141.

Sutton JT, Robertson BC, Jamieson IG 2011. Dye shift: a neglected source of genotyping error in molecular ecology. Molecular Ecology Resources 11: 514-520.

Sutton JT, Robertson BC, Grueber CE, Stanton J-AL, Jamieson IG 2013. Characterization of MHC class II B polymorphism in bottlenecked New Zealand saddlebacks reveals low levels of genetic diversity. Immunogenetics 65: 619-633.

Editorial board member: Kerri-Anne Edge Hill

Received 2 March 2016; accepted 29 June 2016
Sutton JT, Robertson BC, Jamieson IG 2015. MHC variation reflects the bottleneck histories of New Zealand passerines. Molecular Ecology 24: 362-373.

Tallmon DA, Koyuk A, Luikart G, Beaumont MA 2008. onesamp: a program to estimate effective population size using approximate Bayesian computation. Molecular Ecology Resources 8: 299-301.

Taylor SS, Jamieson IG 2008. No evidence for loss of genetic variation following sequential translocations in extant populations of a genetically depauperate species. Molecular Ecology 17: 545-556.

Ujvari B, Belov K 2011. Major histocompatibility complex (MHC) markers in conservation biology. International Journal of Molecular Sciences 12: 5168-5186.

Valkiūnas G 2005. Avian malaria parasites and other haemosporidia. Boca Ratón, CRC Press. 932 p.

Van Oosterhout C, Hutchinson WF, Wills DPM, Shipley P2004. Micro-Checker: software for identifying and correcting genotyping errors in microsatellite data. Molecular Ecology Notes 4: 535-538.

Walsh PS, Metzger DA, Higuchi R 1991. Chelex-100 as a medium for simple extraction of DNA for PCR-based typing from forensic material. BioTechniques 10: 506-513.

Westerdahl H, Hansson B, Bensch S, Hasselquist D 2004. Between-year variation of MHC allele frequencies in great reed warblers: selection or drift? Journal of Evolutionary Biology 17: 485-492.

Wright DJ, Spurgin LG, Collar NJ, Komdeur J, Buke T, Richardson DS 2014. The impact of translocations on neutral and functional genetic diversity within and among populations of the Seychelles warbler. Molecular Ecology 23: 2165-2177.

Yuhki N, O'Brien SJ 1990. DNA variation of the mammalian major histocompatibility complex reflects genomic diversity and population history. Proceedings of the National Academy of Science USA 87: 836-840.

\section{Supplementary Material}

Additional supporting information may be found in the online version of this article:

Table S1. Polymorphic and monomorphic (P/M) microsatellite loci amplified in this study and their corresponding references.

Table S2. Positively selected sites on MHC loci as identified by FEL and REL analyses.

Table S3. Pairwise $F_{\mathrm{ST}}$ values for microsatellite (A), MHC (B), and TLR (C) loci.

Figure S1. Neighbour-Joining tree of 24 identified MHC class II alleles across five South Island saddleback populations.

Figure S2a. Relationships between pairwise $\mathrm{MHC} /$ microsatellite $\left(y=-83.906 x-0.122, R^{2}=0.322, p=0.070\right)$ and
TLR/microsatellite $\left(\mathrm{y}=0.170+0.043, \mathrm{R}^{2}=0.015, \mathrm{p}=0.390\right)$ $F_{\mathrm{ST}}$ values. S2b. Relationship between TLR and MHC loci pairwise $F_{\mathrm{ST}}$ among five South Island saddleback populations $\left(y=-0.005 x-0.002, R^{2}=0.296, p=0.030\right)$.

Figure S3. PhcaTLR1LA1 (SG19) aligned against Gallus gallus toll-like receptor1 (Ref; GenBank Accession NP_001007489) sequence.

The New Zealand Journal of Ecology provides online supporting information supplied by the authors where this may assist readers. Such materials are peer-reviewed and copy-edited but any issues relating to this information (other than missing files) should be addressed to the authors. 